\title{
Interprofessional teamwork: Are we ready for skill assessment?
}

Sandrijn M. van Schaik ${ }^{1}$, Christy Boscardin², Bridget O'Brien², Shelley R. Adler ${ }^{3}$

${ }^{1}$ Department of Pediatrics, University of California San Francisco; San Francisco, CA

${ }^{2}$ Department of Medicine and Office of Research and Development in Medical Education, University of California, San Francisco; San Francisco, CA

${ }^{3}$ Department of Family \& Community Medicine and Osher Center for Integrative Medicine, University of California, San Francisco; San Francisco, CA

Corresponding Author:

Sandrijn van Schaik, MD PhD

Professor of Pediatrics, Division of Pediatric Critical Care

Baum Family Presidential Chair for Experiential Learning

University of California San Francisco

550 16th Street, Floor 5, Box 0106

San Francisco, CA 94143

Phone 415 476-3731

e-mail: Sandrijn.vanschaik@ucsf.edu

\section{Grant support:}

This study was supported by the Stemmler Medical Education Research Fund of the National Board of Medical Examiners.

Dr. van Schaik and Dr. O'Brien received support through Faculty Scholarship Awards from the Josiah Macy Jr. Foundation

Keywords: Teamwork, Interprofessional education, Assessment, Feedback

Sandrijn M. van Schaik MD, PhD, is a Professor in the Department of Pediatrics at the University of California, San Francisco (UCSF), Baum Presidential Chair for Experiential Learning, Director of the UCSF Kanbar Center for Simulation and Clinical Skills and Director of Faculty Development for the UCSF School of Medicine Bridges Curriculum.

Christy Boscardin, PhD, is an Associate Professor in the Department of Medicine and Education Scientist in the Center for Faculty Educators at the University of California, San Francisco

Bridget C. O'Brien, PhD, is an Associate Professor in the Department of Medicine and Education Scientist in the Center for Faculty Educators at the University of California, San Francisco Shelley R. Adler, PhD, is a Professor in the Department of Family \& Community Medicine, Director of the Osher Center for Integrative Medicine, Osher Foundation Distinguished Professor of Integrative Medicine, and Co-Director of the Osher Center NIH T-32 Fellowship Program at the University of California, San Francisco.

\section{Background}


Increasing focus on interprofessional collaboration in the health professions has led to the formulation of competencies in interprofessional collaboration ${ }^{1}$ and widespread implementation of educational efforts to ensure health professionals acquire the associated skills. ${ }^{2,3} \mathrm{~A}$ logical next step is to assess the performance of learners who are the target of these educational efforts in order to verify that they have the required competencies. The number of published teamwork assessment instruments is large and continues to grow. ${ }^{4-6}$ There are several instruments that focus on assessment of individual team members' performance in crisis resource management and other high acuity contexts. ${ }^{7-11}$ There is, however, a relative sparsity of instruments that focus on behavior-based assessment of individual (rather than team) performance in low acuity contexts. We postulated that there likely would be overlap between teamwork behaviors desired in high-acuity and low-acuity settings, but that differences in membership, goals, and tasks of the team would limit the relevance of instruments designed for high-acuity settings. Indeed, our qualitative work among low-acuity healthcare teams confirmed this hypothesis and highlighted the importance of collaboration, respect and attention to team development in low-acuity settings. ${ }^{12}$ In addition, publications documenting the development of existing teamwork assessment instruments most commonly report the use of raters external to the team. It can be argued that determining whether an individual has effective teamwork skills is a judgment best made by others on the team. For these reasons, we set out to develop and validate a instrument to assess individual interprofessional teamwork skills for use in healthcare teams across multiple low-acuity settings that could be used by team members, rather than by external raters. In this paper, we describe the development process of our assessment instrument, discuss the challenges we 
encountered when attempting to validate this instrument, and consider our experience in the context of the literature on interprofessional teamwork skills assessment, evaluation, and feedback.

\section{Instrument Development}

In our instrument development process, we aimed to collect validity evidence applying the unitary view of validity described by Messick, using a framework that includes content, response process, internal structure, relationship to other variables, and consequences. ${ }^{13}$ The first step emphasized validity evidence of content and consisted of qualitative work to explore the constructs underlying interprofessional teamwork in low-acuity settings. The details of this work, which led to the characterization of nine constructs with corresponding observable behaviors, have been published previously. ${ }^{12}$ We translated these behaviors into 25 assessment items (see Appendix and Table 1), which a small team of experts in assessment and interprofessional teamwork reviewed for completeness and relevance. We subsequently pilottested this instrument in 3 sets of interprofessional teams at our institution, chosen to represent both teams of learners and teams of experienced providers in both educational and real-life settings. The first set included 30 teams of students from seven health professions education schools and programs, that participated in an Interprofessional Standardized Patient Exercise (ISPE). During this exercise, which has been described in detail elsewhere,${ }^{14}$ students work in small interprofessional teams (3-4 students) over the course of a half-day session. Students typically do not know their team members prior to the exercise and generally have had limited experience working in interprofessional teams. The second set included 5 teams: 
Integrative Medicine, Palliative Care, Pediatric Bone Marrow Transplant and Women's HIV (2 teams at 2 different campus locations). These teams are well-established, have relatively fixed membership and work together in real-life clinical settings. The third set consisted of 4 teams; these groups included experienced professionals (5-6 per team) who, at the time of study participation, had known each other for 6 months through monthly course sessions in a Continuing Education in Geriatrics course, but did not actually work together in the clinical setting. These teams participated in the same exercise as the students in group 1 just prior to study participation, during the sixth session of their course. All members of all 3 sets of teams were eligible to participate in the study. We recruited team members to the study between January and April 2014. The UCSF Institutional Review Board approved the study.

We imported our assessment instrument and completion instructions into an online survey instrument (Qualtrics ${ }^{\mathrm{TM}}$ ). After the last item, we asked all study participants in the second and third set of teams to provide feedback on the instrument itself by rating the ease of use and usefulness for practice improvement on a 5-point scale (1=not easy/not useful, 5=very easy/useful). In addition, we asked these participants to indicate how long it took to complete the instrument, whether there were any items that were difficult to answer, and, if so, why. We informed all prospective study participants that their ratings would remain anonymous and would not be shared with the individuals whose skills they assessed. This information was reiterated in the introduction to the online instrument. To ensure anonymity, we assigned all participants a unique study ID and asked them to indicate the profession (or professional 
school) of the person they rated only; we did not collect data on the profession (or professional school) of the person who completed the instrument.

\section{Challenges encountered}

Despite the fact that we included a variety of teams in our study, comprising both novices and experienced healthcare professionals, and that assessments were anonymous and ratings were not shared with those who were assessed, we found that study participants consistently rated their team members' teamwork skills at the higher end of the scale. This finding appeared in all 3 sets of teams, even with a large number of raters $(n=143)$ and high completion rates among participants.

The 143 raters in our study (94 students, 49 practicing health care professionals) completed the instrument on 170 of their team members, with an average of 3 ratings per rater, for a total of 397 completed instruments. Mean ratings across all team sets were between 4.5 and 4.9 on a 5-point scale, with 1 indicating that the rater strongly disagreed that the rated individual exhibited a certain behavior and 5 indicating strong agreement (Table 2). A more granular look at the data revealed that ratings of 1-2 (indicating low performance) were rare across the data set. Among students, there were no ratings of 1 and only 10 ratings of 2 across all items and all raters ( $0.2 \%$ of all ratings). Among experienced providers, there were 5 ratings of 1 and 55 ratings of 2 ( 0.2 and $1.6 \%$ of all ratings, respectively). This limited spread in data prohibited meaningful statistical analysis to further establish validity evidence based on internal structure (i.e. factor analysis). Of note, we found good validity evidence based on response process, 
which is essentially integrity of the data, ${ }^{13}$ since the proportion of missing data was small: across the entire data set, there were 86 blank responses and 150 "unable to answer" responses; out of 9,925 total (2.4\%). In addition, study participants rated the instrument's ease of use as high, with a mean rating of 4.56 (5-point scale, range 4 to 5). Participants were more divided about the usefulness of the instrument for practice improvement, with a mean rating of 3.6 (range 2-5). The reported average time for completion was 7 minutes, with a range of 2-10 minutes.

\section{Discussion}

We discovered during the instrument development process that a rigorous approach to instrument development by itself does not guarantee a valid instrument, at least not based on commonly accepted standards demonstrating evidence for validity. ${ }^{13}$ Despite some evidence for content validity and response process, we were not able to collect validity evidence for internal structure. As a result, collecting data on the remaining two components of Messick's framework, relationship to external variables and consequences, would have been without meaning. On reflection, we recognize that the perspectives and skills of raters, the nature of what is being rated, and the context and culture in which ratings occur are all important factors that can greatly influence the validity of an assessment instrument.

There are several potential explanations for our failure to collect complete validity evidence. Perhaps all the people who were rated in this study truly excelled in teamwork, resulting in a study population too uniform to allow for any variability in study data. This explanation seems 
highly unlikely, however, considering the fact that the 3 sets of teams in our study were quite different from one another in terms of experience with teamwork. A second possibility is that the raters did not understand how to use the instrument effectively, since none of them received formal training in using the instrument. Rater training may have provided different results, although the literature suggests that the effect of rater training is limited. ${ }^{15}$ Moreover, if rater training is essential for effective use of an instrument, its acceptability for healthcare teams with limited time to work on practice improvement will likely be low. A third, related, explanation may be that raters had an inaccurate or imprecise understanding of the constructs underlying the assessment instrument. ${ }^{16}$ Since explicit teamwork training is still relatively new in health professions education, the raters in our study may not have had a clear frame of reference for behaviors associated with low and high team member performance. A fourth, and in our opinion most likely, explanation lies in the culture of healthcare, where providing feedback, particularly feedback focused on the domain of interpersonal and communication skills, remains a complicated endeavor. Several studies have shown that so-called generosity or leniency bias is common in medical education, ${ }^{16-20}$ leading to higher ratings than what would be reasonably expected.

The reasons for this generosity bias are likely complex and multi-faceted. We documented similar generosity bias in a prior study in which a different cohort of students in the ISPE program at our institution provided written feedback for their team members. ${ }^{21}$ Students in that study typically provided fairly generic, positive comments, such as "great job." In subsequent focus group discussions, we uncovered that fear of being seen as too critical played 
a major role in the learners' positive assessments. In addition, the students struggled more with giving critical feedback on teamwork skills than on history taking skills and seemed to conflate the former with personality traits. This phenomenon is akin to that described by Ginsburg and colleagues in their discussion of evaluation of professionalism, which typically addresses character traits of people rather than specific behaviors. ${ }^{22}$ Interestingly, while the students in the ISPE teams were hesitant to provide constructive feedback, they did indicate desire for more critical feedback. To illustrate how deep-seated the tendency to "be nice" is, these same students, in an anonymous survey, rated the feedback they received from their team members as highly useful. ${ }^{23}$ One important factor that may drive this tendency is the need for an established relationship to provide effective feedback. ${ }^{24}$ This relationship may be necessary to create a context in which it feels safe to deliver critical feedback, but could also be needed because people may hesitate to make judgments based on limited interactions and observations. The students in the ISPE teams did not have an established relationship, since most of them met for the first time at the beginning of the exercise, which lasted only a half day. Conversely, it can also be argued that having a relationship might make people less willing to be critical. We noted this in 2 separate studies about interprofessional feedback in which residents expressed hesitance to provide constructive feedback to nurses out of fear that it would damage their relationship. ${ }^{25,26}$ This may be related to the interprofessional context as a result of complex interprofessional dynamics, although reluctance to give critical feedback has been described within the medical profession, as well. ${ }^{27}$ In the current study, we did not find any difference in ratings between well-established teams (whose members, presumably, have developed relationships) and teams with shorter life spans. We collected all data anonymously 
and explicitly told participants that we would not share their ratings with the team members they assessed. Theoretically, this should have created an equally safe context for critical ratings across all teams, but differences in ratings based on frequency and duration of interactions would have been expected.

This is not the first instrument that is struggling with collecting validity evidence due to generosity bias. An instrument to assess physician-in-training leadership skills, developed by a multi-institutional team that two of the authors (SVS and CB) were part of, also suffered from high ratings with limited variability (unpublished data). Also, in a report describing the development of the iTOFT, an instrument with an intent and development process similar to our own, the authors note that "the Likert scale did not work well," but do not elaborate on reasons or provide any further data. ${ }^{28}$ We are left with the question of whether we should even try to measure individual teamwork skills. Perhaps this and similar domains that touch on interpersonal and communication skills are too complex to disentangle and reduce to discrete items. These teamwork behaviors may be better explored through qualitative assessment approaches, which, when well-designed, have demonstrated good evidence of validity, although they are labor intensive. ${ }^{29}$ Qualitative assessment instruments are, however, unlikely to overcome issues with generosity bias, as we noted in prior studies in which students wrote narrative comments. Perhaps we should shift the focus primarily to formative assessment that can increase reflective practice, rather than trying to capture skills in numeric format to determine level of competence. 
Conclusion: Despite the emphasis on interprofessional collaboration and competency assessment in healthcare, assessment of interprofessional teamwork skills is not easily accomplished using traditional psychometric approaches. Employing alternative approaches such as qualitative assessments may be more feasible, but does not resolve the fundamental issue of the culture of feedback in medicine and medical education: the fear of appearing too critical and the tendency to provide praise exclusively. This cultural patterning is the biggest challenge to using feedback and assessment for performance improvement, both in the intraand interprofessional realms. Deeper exploration into the forces that maintain this culture and experimentation with approaches that might help overcome existing barriers to critical feedback should be prioritized over adding additional instruments.

\section{Acknowledgments:}

The authors wish to thank all of the team members who generously donated their time to the study.

\section{Ethical approval:}

The UCSF Institutional Review Board approved the study.

\section{Declaration of interest:}

The authors declare no conflicts of interest. 


\section{References}

1. Core competencies for interprofessional collaborative practice: Report of an expert panel. Washington DC: Interprofessional Education Collaborative Expert Panel; 2011.

2. Abu-Rish E, Kim S, Choe L, et al. Current trends in interprofessional education of health sciences students: A literature review. J Interprof Care. 2012;26(6):444-451.

3. Olson R, Bialocerkowski A. Interprofessional education in allied health: a systematic review. Med Educ. 2014;48(3):236-246.

4. Valentine MA, Nembhard IM, Edmondson AC. Measuring teamwork in health care settings: a review of survey instruments. Med Care. 2015;53(4):e16-e30.

5. Havyer RD, Wingo MT, Comfere NI, et al. Teamwork assessment in internal medicine: a systematic review of validity evidence and outcomes. J Gen Intern Med. 2014;29(6):894.

6. Valentine M, Nembhard I, Edmondson A. Measuring teamwork in health care settings: a review of survey instruments. Harvard Business School Technology \& Operations Mgt Unit Working Paper. 2012(11-116).

7. Kim J, Neilipovitz D, Cardinal P, Chiu M, Clinch J. A pilot study using high-fidelity simulation to formally evaluate performance in the resuscitation of critically ill patients: The University of Ottawa Critical Care Medicine, High-Fidelity Simulation, and Crisis Resource Management I Study. Crit Care Med. 2006;34(8):2167-2174.

8. Malec JF, Torsher LC, Dunn WF, et al. The Mayo high performance teamwork scale: reliability and validity for evaluating key crew resource management skills. Simulation in Healthcare. 2007;2(1):4-10. 
Interprofessional teamwork assessment

9. King H, Battles J, Baker DP. TeamSTEPPS ${ }^{\mathrm{TM}}$ : Team strategies and tools to enhance performance and patient safety. In: K H, Battles J, Keyes M, eds. Advances in Patient Safety: New Directions and Alternative Approaches. Vol 3. Rockville (MD): Agency for Healthcare Research and Quality; 2008.

10. Frankel A, Gardner R, Maynard L, Kelly A. Using the communication and teamwork skills (CATS) assessment to measure health care team performance. Joint Commission Journal on Quality and Patient Safety. 2007;33(9):549-558.

11. Fletcher G, Flin R, McGeorge P, Glavin R, Maran N, Patey R. Anaesthetists' Non-Technical Skills (ANTS): evaluation of a behavioural marker system †. Br J Anaesth. 2003;90(5):580588.

12. Schaik SM, O'Brien BC, Almeida SA, Adler SR. Perceptions of interprofessional teamwork in low-acuity settings: a qualitative analysis. Med Educ. 2014;48(6):583-592.

13. Messick S. The psychology of educational measurement. Journal of educational measurement. 1984;21(3):215-237.

14. Wamsley M, Staves J, Kroon L, et al. The impact of an interprofessional standardized patient exercise on attitudes toward working in interprofessional teams. J Interprof Care. $2012 ; 26(1): 28-35$.

15. Williams RG, Klamen DA, McGaghie WC. Cognitive, social and environmental sources of bias in clinical performance ratings. Teach Learn Med. 2003;15(4):270-292.

16. McGill DA, van der Vleuten CPM, Clarke MJ. Supervisor assessment of clinical and professional competence of medical trainees: a reliability study using workplace data 
and a focused analytical literature review. Advances in Health Sciences Education. 2011;16(3):405-425.

17. Dudek NL, Marks MB, Regehr G. Failure to fail: the perspectives of clinical supervisors. Acad Med. 2005;80(10):S84-S87.

18. Bandiera G, Lendrum D. Daily encounter cards facilitate competency-based feedback while leniency bias persists. CJEM. 2008;10(01):44-50.

19. Archer J, McGraw M, Davies H. Assuring validity of multisource feedback in a national programme. Arch Dis Child. 2010;95(5):330-335.

20. Speer AJ, Solomon DJ, Fincher R-ME. Grade inflation in internal medicine clerkships: results of a national survey. Teach Learn Med. 2000;12(3):112-116.

21. Mandal J, Avdagic K, Wamsley M, Brock T, van Schaik S. Beyond "great job": Feedback among students on interprofessional teams. Journal of Interprofessional Education \& Practice. 2016;5:37-43.

22. Ginsburg S, Regehr G, Hatala R, et al. Context, conflict, and resolution: a new conceptual framework for evaluating professionalism. Acad Med. 2000;75(10):S6-S11.

23. van Schaik SM, Regehr G, Eva KW, Irby DM, O'Sullivan PS. Perceptions of peer-to-peer interprofessional feedback among students in the health professions. Acad Med. 2016;91(6):807-812

24. Telio S, Ajjawi R, Regehr G. The "educational alliance" as a framework for reconceptualizing feedback in medical education. Acad Med. 2015;90(5):609-614. 
25. Vesel T, O'Brien B, Henry D, van Schaik S. Residents' perceptions of interprofessional feedback2014. Located at: Association of American Medical Colleges Medical Education Meeting, Chicago, IL.

26. van Schaik S, Plant J, O'Brien B. Challenges of interprofessional team training: a qualitative analysis of residents' perceptions. Education for Health. 2015;in press.

27. Sargeant J, Mann K, Sinclair D, Van der Vleuten C, Metsemakers J. Understanding the influence of emotions and reflection upon multi-source feedback acceptance and use. Advances in Health Sciences Education. 2008;13(3):275-288.

28. Thistlethwaite J, Dallest K, Moran M, et al. Introducing the individual Teamwork Observation and Feedback Tool (iTOFT): Development and description of a new interprofessional teamwork measure. J Interprof Care. 2016;30(4):526-528.

29. Cook DA, Kuper A, Hatala R, Ginsburg S. When assessment data are words: validity evidence for qualitative educational assessments. Acad Med. 2016;91(10):1359-1369. 